\title{
Exploring the Effects of Real-Time Hologram Communication on Social Presence, Novelty, and Affect
}

\author{
Michael Mazgaj, MBA \\ University of Nebraska \\ Omaha \\ mjmazgaj@unomaha.edu
}

\author{
Alexis d'Amato \\ University of Nebraska \\ Omaha \\ adamato@unomaha.edu
}

\author{
Joel Elson, $\mathrm{PhD}$ \\ University of Nebraska \\ Omaha \\ jselson@unomaha.edu
}

\author{
Douglas Derrick, $\mathrm{PhD}$ \\ University of Nebraska \\ Omaha \\ dcderrick@unomaha.edu
}

\begin{abstract}
With the COVID-19 social distance orders, multichannel technologies are more critical than ever for collaborating. These technologies offer psychological benefits that improve user and collaborative experiences. The main objective of this research was to explore hologram communication within a telecommunication context. This research looked to understand the effect of hologram communication on affect (i.e., emotion) and studied the relationship between holograms and social presence, and how the sense of being together may change or amplify positive messages. This paper focuses specifically on how social presence and emotional responses differ between hologram communication and video communication, such as Zoom or Google Hangouts. We used a novel hologram projection system and conducted an experiment with 98 people and found that hologram technology improved the perception of co-presence and affected two dimensions of novelty. Additionally, there were some indications of other findings that were less compelling. However, this provides further insight into the context of $3 D$ communication for collaboration.
\end{abstract}

\section{Introduction}

Everyone uses technology to communicate. Whether it is a phone call across the country, a video conference for work, or an online workout class, technology has changed the way humans work and live. Technology does not always mirror face-to-face interactions, but it attempts to create the same connection when physically distant.

Communication methods and technologies progress throughout history, enabling more productive collaboration and surpassing physical boundaries [9]. During the COVID-19 pandemic, this type of productive communication is essential to enable meaningful connections. Technology, such as Zoom and Facetime, bring people together and facilitate collaboration. Digital media and technologies have dominated many research and development laboratories across universities and private institutions to understand the psychology and the effects these technologies have on humans.

Computer-mediated communication (CMC) is a relatively new field that assists in progressing understanding of communication through digital media and technology. CMC involves instant messaging, shared hypermedia, blogs, graphical charts, and other media [23].

Computer-mediated communication (CMC) refers to "any human communication achieved through computer technology" $[19 ; 13]$. CMC allows people to communicate despite their physical distance, aiding distance learning, work from home, and international business. It simultaneously enables people to be connected and mobile, thus changing the way humans work and play $[8 ; 15]$.

However, different types of CMC mediums limit one's ability to communicate effectively due to the lack of non-verbal cues or feedback when communicating. This limitation is explained by media richness theory $[8 ; 6]$. Media richness theory examines the medium as constraints on the ability to express nonverbal emotional-relational communication [25]. Face-to-face communication is the richest form of communication, offering lots of non-verbal cues and feedback, while written text in an email or text message is the leanest, leaving the meaning up for interpretation [6].

Media richness can help one communicate more effectively and can change how one feels [8;6]. Each CMC technology has a different effect on the people who use it (e.g., how connected they feel to others). $\mathrm{CMC}$ has evolved to the extent of mainstream use of two-dimensional (2D) video communication; however, communication technology has yet to 
progress beyond the constraints and efficiencies of 2D technology. The underlying problem within new CMC technologies is what type of psychological effects are they having on their users while communicating. Holograms are one of the latest advances in CMC and this paper provides an initial understanding if hologram technologies are a justifiable addition to $\mathrm{CMC}$ and why.

\section{Hologram Technology for Collaboration}

Three-dimensional projection is a new medium for communication. With the start of the 21st Century, the technology for creating 3D or "holographic images" has seen much improvement. Developing technology to produce 3D images has been an arduous task among physicists, engineers, opticians, and other disciplines. After further research around lasers, optical holography was a method to retrieve the likeness of a subject from its unfocused diffraction pattern [6]. With the onset of computer science as a discipline, digital holography has started to surface as a realm of possibility. Digital holography is the same process as Optical Holography with wave reflection and storage. However, digital holography involves electronically recording and storing within a computer [27]. With this innovation, the questions of utilizing digital holography as a medium of $\mathrm{CMC}$ are abundant and unanswered. Specifically, what are the theoretical constructs that can be used to explore the benefits of hologram communication, and does the effort of producing the images enhance communication. These unknowns act as strong motivations to develop research and understanding of 3D CMC technology. It may be expected that a hologram is creating easy and better ways to communicate. The following sections propose potential constructs that might explain the benefit of holographic collaboration.

\section{Theoretical Background}

The major theoretical concepts include various facets of novelty, media richness, core affect, and presence.

\subsection{Novelty}

With the onset of digital media and its acceleration of efficiencies, there are still many unknowns around technology and communication. One major limitation within this research endeavor is that of "technology novelty." Technology novelty or "technology risk" is defined as the newness, to the development organization, of the technologies used within product development [29]. Arguably, technology adoption and use may fade as the novelty wears off. This attrition outcome becomes difficult when measuring the effects of any new technology.

Digital holography is simply no exception to how novelty may determine its future applications. One major setback for the digital holography movement was the death of the 3D television. In addition, two obstacles such as the lack of 3D content from live TV and the onset of smart TV laden with applications, such as Netflix, saw 3D personal entertainment rise and fall quickly [20]. The redesign of the traditional 2D home television into a 3D was an overshoot, but not far from practical applications of holography.

As technology is advancing, humans are progressing with it, additionally humans using holography is novel. In the case of the 3D televisions, the novelty wore off, and the application of entertainment rebounded back to 2D technology. Regarding this research, novelty is considered a fundamental characteristic that describes traits that are "new" or "fresh" of an innovation within the perspective of the user [31]. Research around novelty is user-focused, as having a technology available is simply not enough for accepted use [1]. This research will focus on understanding an individuals' novel experience: if a technology is "refreshing," and the "newness" of communicating in different ways.

\subsection{Media Richness Theory}

Originally, media richness theory was developed by Lengel et al [15]. Seeing medium characteristics as constraints on one's ability to communicate nonverbal, emotional, and relational communications [15]. These constraints exist within four (4) areas: nonverbal, natural language, feedback, and personal focus.

The nonverbal constraint is the level to which a person understands physical gestures, vocal tones, vocal inflections, and eye contact, measuring one's ability to convey information without a coherent lingual meaning. Natural language is the spoken language that moves conceptual ideas and coherent information to one another, measuring the use of formal and informal grammar, punctuation, fluency, slang, verbal play, and sentence structure. The feedback constraint measures a medium's ability to facilitate immediate feedback or the rate of reaction from the receiver of the message. Many CMC mediums experience high levels of constraining immediate feedback. Lastly, personal focus is the constraint that facilitates emotions and subjective beliefs, which affects the way the message is expressed and interpreted. This constraint is very 
important, as it encompasses human emotions around each other and through the $\mathrm{CMC}$ medium being utilized [25].

These constraints are once piece of the puzzle to understanding the limitations and strengths of current and future $\mathrm{CMC}$ technologies. One concept worth mentioning is that of "media naturalness." This concept is important to note, as it views communication as a dynamic process that changes over time through an evolutionary process [13]. With the failure of $3 \mathrm{D}$ personal TVs, media naturalness may explain that humans were not ready to experience 3D images within an entertainment scope. However, with the current trending applications within the current market, humans may be ready to experience 3D telecommunication.

\subsubsection{Media Richness Continuum}

To conceptualize the measurement of media richness across technologies, the medium richness continuum was created. This model identifies the previous constraints as categories compared to the type of communication used and determining how "rich" or "lean" it may be. [15]. Sherblom [25] continues to describe the varying levels within a media richness continuum; within this continuum the types of interaction affecting identity.

Table 1. Media Richness Continuum [26]

\begin{tabular}{|l|c|l|l|l|l|}
\hline \multicolumn{6}{|l|}{ Media Richness Continuum } \\
\hline Constraints & Medium & $\begin{array}{l}\text { Personal/Nonverbal } \\
\text { Cues }\end{array}$ & $\begin{array}{l}\text { Immediate } \\
\text { Feedback }\end{array}$ & $\begin{array}{c}\text { Natural } \\
\text { Language }\end{array}$ & $\begin{array}{l}\text { Personal } \\
\text { Emotions }\end{array}$ \\
\hline Rich & Face-to-Face & Available & Available & Available & Available \\
\hline \multirow{4}{*}{$\downarrow$} & Telephone & $\begin{array}{l}\text { Lacks Facial } \\
\text { Expression, Physical } \\
\text { Gestures }\end{array}$ & Available & Available & Available \\
\cline { 2 - 6 } & Personal Text & Specialized, Limited & Available & $\begin{array}{l}\text { Specialized, } \\
\text { Limited }\end{array}$ & $\begin{array}{l}\text { Somewhat } \\
\text { Limited }\end{array}$ \\
\cline { 2 - 6 } & Email & More Limited & $\begin{array}{l}\text { Less } \\
\text { Immediate }\end{array}$ & $\begin{array}{l}\text { More } \\
\text { Formal }\end{array}$ & $\begin{array}{l}\text { Less } \\
\text { Common }\end{array}$ \\
\hline Lean & $\begin{array}{c}\text { Impersonal } \\
\text { Written } \\
\text { Memo/Report }\end{array}$ & Unavailable & Unavailable & Unavailable & Unavailable \\
\hline
\end{tabular}

The continuum addresses constraints within the medium and assists in visualizing the effects of the constraints along with current $\mathrm{CMC}$ technologies. The richest form of technology is face-to-face communication; the mainstream CMC technology for this is $2 \mathrm{D}$ video and audio calls. So, the notion of a holographic call and its media richness comes into question; however, to understand how a holographic call may work, it is important to understand the concept of holography.

Within CMC literature, one of six conceptualizations of presence are considered "presence as social richness." Described mainly as the connection between social presence theory and media richness theory, this facet identifies the extent to which a medium is perceived as sociable, warm sensitive, personal, or intimate when it is utilized to interact with other people [16]. This is the facet within presence to be measured in this study. Previous studies with this narrow of a context identify social, intuitive, and emotional tasks are difficult to analyze. For example, electronic mail (email) is a lean form of media richness that is less appropriate for tasks requiring social presence and reported positive outcomes associated with their use of email [21]. Displaying how the relationship between social presence and emotions may be dependent on a social, intuitive, and emotional tasks. Yet, Rice et al [21] did support that there is a relationship between social presence and performance, and how CMC facilitated spanning across face-toface, video, audio, and written forms of communication.

\subsection{Core Affect \& Emotions}

The measurement of emotions and affect requires understanding the theory of emotions. Basic emotions have an innate ability to create universal expressions and unique feelings of motivation [11]. For organisms such as humans, emotions are an important motivation framework. Emotions drive behavioral reactions to environmental and internal occurrences; often dictating needs and goals [5]. This is of significance, as it builds the foundational understanding of how emotions affect ones' psychological state around communicating in different media.

With basic emotions possessing a combination of social and biological mechanisms, the concept of core affect may build an easier framework to tie to social presence. Core affect is a neurophysiological state consciously accessible as the simplest raw (nonreflective) feelings evident in moods and emotions [24]. Being the "simplest feelings," core affect may not be reduced to anything simpler at a psychological level. This concept does not examine the neurophysiological, rather the psychological instead. To explain the concept of core affect further, it is the simplest accessible state of consciousness as a single simple feeling of good or bad, being "emotion," or feeling energized or enervated, being "mood." Emotion changes rapidly accompanied by cognitions, physiological changes, and behaviors. Mood identifies a prolonged experience with behavioral demeanor, thoughts, and motivations. Even though core affect may fully not explain emotion or mood, it is a key ingredient within both [32]. Literature suggests using dimensional structures of mood and emotion such as the 12-Point Circumplex Model; however, this research endeavor requires to focus on mainly affective emotional states such as "general positive affect," and "general negative affect." Core affect is 
important at a simple level, as it has literature and metrics assists in furthering our understanding of emotions. Emotions and emotional characteristics are structured differently across individuals. An individual's affective reaction to an experience is maybe measured across their valence, arousal, and dominance [4].

A central characteristic of emotions is that of its valence. Valence identifies whether an emotion is pleasant or unpleasant [8]. With experiences of communication through technology, it is important to understand the degree to which positive or negative emotions may range. Valence may assist in an additional measurement of positive and negative emotion when communicating through a 3-D technology versus a 2-D technology. In addition to valence, arousal is additionally important to identify the degree of excitement in an individual. Varying from "excited" to "calm," Arousal assists in understanding the energy of the emotion behind it [4]. Within this research context, arousal is important because it signals the depth of an emotion and if it is felt.

\subsection{Presence}

Presence refers to one being immersed within a medium $[7 ; 15]$. Presence is felt towards a character in a novel or a friend over text. Presence is also felt when communicating via technology [14] when using a cell phone, social media, or computer-conferencing [15].

The concept of "presence" holds multiple typologies that are specific to experiences within CMC mediums (e.g., telepresence, mediated presence, spatial presence, physical presence, etc.). Presence is important to understand at a basic level because it measures the feeling of all mediated experiences, from reading a novel to riding an immersive virtual reality (VR) simulator. Technologies attempt to produce larger feelings of presence by capturing human sensory channels $[7 ; 14]$. To use presence as an indicator in telecommunications is limited, though, because it may depend on the immersive experience of the technology. Sensory channels within mainstream telecommunication technologies are limited to audio and sight, leaving out other human senses such as smell, touch, and taste.

\subsubsection{Social Presence \& Co-Presence}

Described as the feeling of psychological proximity, closeness, connectedness, and intimacy with another person [28], social presence has the potential to become very prominent within telecommunication technology. Facebook is seen as one of the most popular social network platforms due to their use of social presence. This result are from a concept within social presence known as co-presence.

Co-presence expands the feeling that the user's perception and feeling is mutual experiencing the same emotional closeness [26]. A more robust definition of social presence is containing a technology and psychology requirement, a central focus on mediating social interaction, and measurable conditions [2; 3]. These concepts may facilitate empathetic emotions ultimately leading to better

Figure 1. Perceived Symmetry Analysis [9]

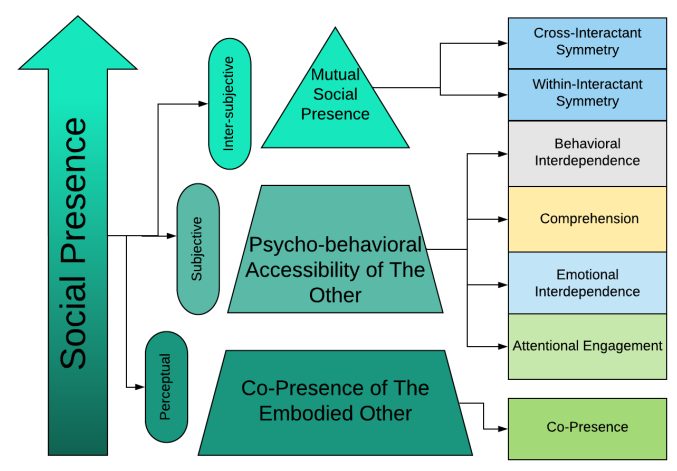

communication [19].

As displayed in Figure 1, The Networked Minds Inventory measures multidimensional on mutual awareness and mutual understanding. The first level is perceptual, which identifies co-presence and the degree to which users feel aware or detected in the same space. The second level is subjective: the degree to which the other appears to interact with each other, focusing on verbal and physical behaviors. The third level is mutual social presence. This occurs when comparing feelings between one's self and the other.

Within level two, there are four sub-facets of psycho behavioral accessibility: attentional engagement, emotional interdependence, comprehension, and behavioral interdependence. Attentional engagement measures attention used in mediated environments; emotional interdependence are the transfer of emotional interaction at observable times; comprehension measures what is being understood while being communicated; and behavioral interdependence measures responses to behavior, such as mimicry, yawning, or patterns of taking turns talking. Level three sub-facts measure "perception of self," and "perception of other" [9].

The Networked Minds Inventory is built to examine two individuals. Figure 1 gives a visual representation of how the perceptions of "self" and "other" are correlated as perceived symmetry. Adaptations of methodologies allow the application of different research contexts. Later sections will discuss 
these methods and steps to adapt them to build context around the original research question to address the concepts of social presence, emotions, and novelty.

Face-to-face interactions occur in a threedimensional space, allowing for high media richness and high social presence, and hologram technology attempts to imitate this. The relationship between the concepts of social presence and emotions may be a more promising endeavor. The existing relationship of personal emotions within CMC is typical within "rich" types of mediums. This is due to the availability within the particular constraint, as well as those of immediate feedback, natural language, and personal/nonverbal cues. The purpose of this research is to understand the relationship between social presence, emotion, and novelty in computer and hologram communication. Because media richness varies between technology mediums, we explored if those changes can alter the affect and social presence felt by users. The research questions for this study are:

1. What is the relationship between video and hologram communication and social presence?

2. How is affect related to video versus hologram communication?

3. How is novelty related to video versus hologram communication?

\section{Research Study}

The main objective of this research was to understand the relationships between social presence, changes in emotion, and novelty across two CMC technologies: 2D video and audio, and 3D hologram and audio. 2D videoconferencing is a popular conventional CMC used frequently. Utilizing this technology as a condition assists in determining how concepts relationships may change with the new use of 3D CMC and to what extent.

\subsection{Procedure}

A flow of the study is shown in Figure 2.

Figure 2. Study design

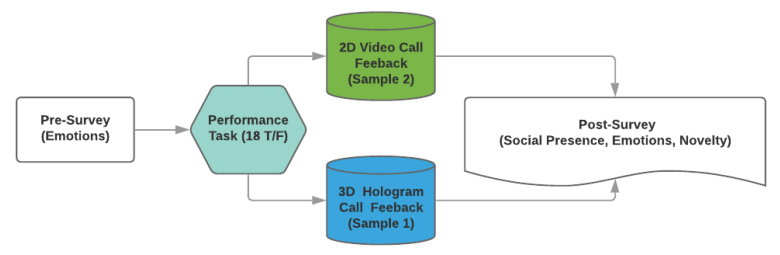

Participants were assessed with the Watson \& Clark's [30] PANAS-X as part of a pre-survey to establish a baseline of positive and negative affect. Similar to the PANAS, the PANAS-X scale measures the general dimension, basic negative emotions, basic emotions, and other affective states. Additionally, to provide valence, arousal, and dominance domain, the Self-Assessment Manakin (SAM) was given in the post-survey [10].

At the conclusion of the pre-survey was an 18question true/false assessment was established as a performance task for participants to receive feedback. The questions were meant to involve critical thinking and creative thought. Following the pre-survey, participants were randomly assigned to one of either the 2D or 3D CMC condition for a feedback session. This feedback session involved a confederate telling the participant that they achieved a high score on the questionnaire and was designed to elicit positive emotion. The feedback session was scripted and went through a manipulation check by approximately 11 graduate and undergraduate.

Social presence was evaluated within the postsurvey. The Networked Minds Inventory was adapted to facilitated one-way and measure how an individual feels immediately after the feedback session to evaluate the degree of social presence of the CMC technology condition. The questionnaire to measure the different underlying constructs within the postsurvey using an adapted version from Biocca et al [3]. An additional consideration with this study was the effect of novelty, and how the user may react to a 3D video call, as it is a very new experience. Novelty is discussed more as a limitation in the study, as the concept can vary depending on the context of the individual and their experiences. To assess novelty of the participant's 3D communication across the CMC technologies, a simple 3-item questionnaire that was adapted to the CMC technologies from Wells et al [31]. Furthermore, it was adapted to be a 5-point Likert Scale. The adapted questionnaire explores measurements of how novel the experience to a user, how "new" or "refreshing" the technology was, and if the form of communication was novel to them.

\subsection{Apparatus}


To assess and compare concepts across the different conditions, we built several LVAH kiosks that are capable of creating a real-time 3D digital projection of a Google Hangouts Call. To allow flexible data collection, a desktop computer with an IntelRealSense camera and Logitech headset were used to administer real-time feedback.
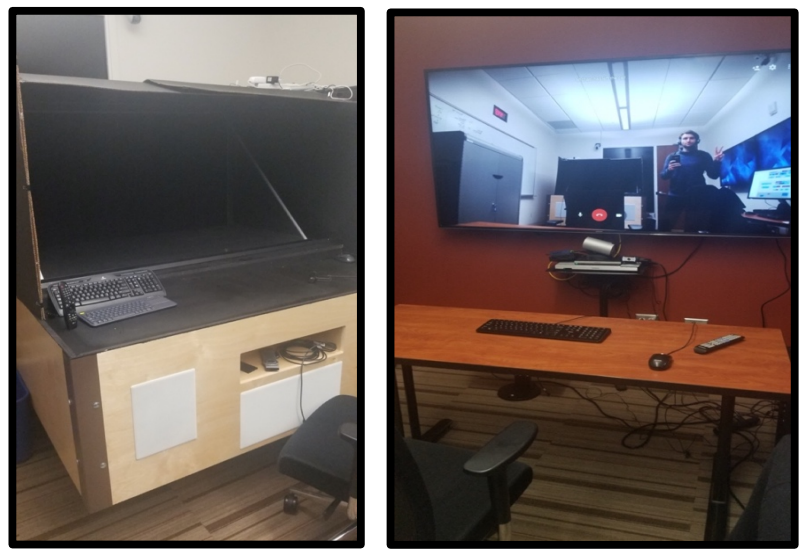

\subsection{Sample}

The experiment used ninety-eight (98) participants between the ages of 19 to 49 with the average age of approximately 22-23 years of age. Of the 98 participants, 42 were male and 56 were female. Refer to Table 2 for the overall sample demographics of the study and conditions.

Table 2. Sample demographics

\begin{tabular}{|l|c|c|}
\hline & $\begin{array}{c}\text { 2D Condition } \\
\text { Participants }\end{array}$ & $\begin{array}{c}\text { 3D Condition } \\
\text { Participants }\end{array}$ \\
\hline Number Male & 20 & 22 \\
\hline Number Female & 28 & 28 \\
\hline Minimum Age & 19 & 20 \\
\hline Maximum Age & 41 & 49 \\
\hline Average Age & 22 & 22.74 \\
\hline
\end{tabular}

Participants were students from the College of Business Administration. This sample was targeted with the assumption that the participants learn a combination of technology and communication skills that are necessary to enter the workforce.

\section{Results}

To ensure that adapted questionnaires were measuring the same concept, the questions' Cronbach alpha was used to ensure the reliability of internal consistency. Cronbach [7] developed the alpha to understand if the items were measuring the same concept or construct; expressed between 0 and 1 . With 0 showing low internal constancy and 1 showing high internal constancy, the adapted questionnaires of social presence, emotions, and novelty were analyzed with this metric.

With multiple facets within social presence, it was necessary to find the internal consistency of the subgroups. The adapted questions within the subgroups are 'co-presence,' and 'psycho-behavioral accessibility.' These constructs showed high internal consistency, as the Cronbach's alphas for copresence was 0.87 , psycho-behavioral accessibility was 0.83 , emotional interdependence was 0.79 , comprehension was 0.76 , and behavioral interdependence was 0.81 .

Regarding emotions, the adapted PANAS-X scale used data to measure two constructs: general positive affect and general negative affect. The construct general positive affect had high internal consistency, as the Cronbach's alphas for the pre-survey was 0.91 and 0.92 for the post-survey. The construct general negative affect also had high internal consistency, as the Cronbach's alpha for the pre-survey was 0.85 and 0.81 for the post-survey. The same questionnaire was used twice to establish what has changed in emotion after the task and feedback session. Data used from the scale focused on attempting to understand significant changes in means from the 10-question general positive affect questions.

Lastly, the construct of 'novelty' showed high internal consistency as determined by Cronbach's alpha of 0.88 .

\subsection{Effects on Social Presence}

The initial objective was to understand the degree of social presence from the user's perspective. We used Networked Minds Inventory to understand the sense social presence between the video call and hologram call conditions. Accordingly, we conducted a within-interactant subjective symmetry analysis. Correlation of values of the perception of self and perception of other were measured. Within-Interactant Symmetry values ranging closer to " 0.00 ," are considered weak correlation coefficients, and values closer to " 1.0 " being a strong correlation coefficient. After correlations values were found, we performed a Fischer Z-Transformation which allow us to transform the symmetry correlation values of "r," to a $Z$ score by which we can test significance. The results of this analysis show that overall, social presence did not differ between the video call and hologram call. Only the measurement of "co-presence" proved to be significant between the two conditions.

Table 3. Networked Minds Inventory

\begin{tabular}{ccc}
$\begin{array}{c}\text { Video } \\
\text { call }(z)\end{array}$ & $\begin{array}{c}\text { Hologram } \\
\text { call }(z)\end{array}$ & $p$ \\
\hline
\end{tabular}




\begin{tabular}{llcc}
\hline $\begin{array}{l}\text { Behavioral } \\
\text { Independence }\end{array}$ & 0.91 & 0.86 & 0.08 \\
$\begin{array}{l}\text { Comprehensive } \\
\text { Construct }\end{array}$ & 0.66 & 0.59 & 0.14 \\
$\begin{array}{l}\text { Emotional } \\
\text { Interdependence }\end{array}$ & 0.82 & 0.89 & 0.08 \\
$\begin{array}{l}\text { Attentional } \\
\text { Engagement }\end{array}$ & 0.54 & 0.57 & 0.40 \\
Co-Presence & 0.22 & 0.90 & $0.001^{* *}$ \\
\hline
\end{tabular}

The graphs that show the symmetry co-relations for co-presence measurement for the hologram and video calls are shown in the figures below. As is shown, the degree of co-presence symmetry is much stronger in the hologram rather than the video call. Likewise, there are other indicators that show that Behavioral Independence and Emotional Interdependence are affected. With behavioral interdependence being greater in video and emotional interdependence being greater in the hologram call.

Figure 3. Co-Presence symmetry for hologram call Graph of Within-Interactant Symmetry for Hologram

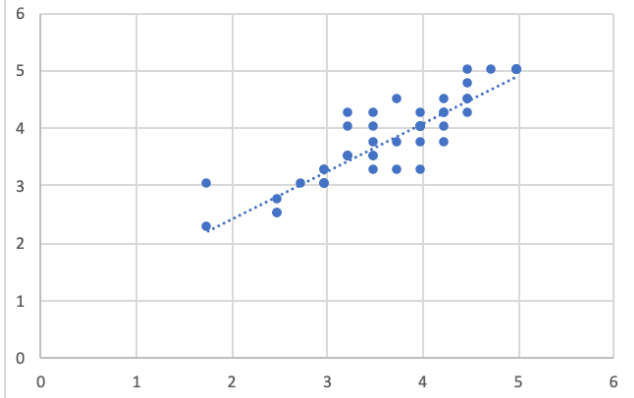

Figure 4. Co-Presence symmetry for video call

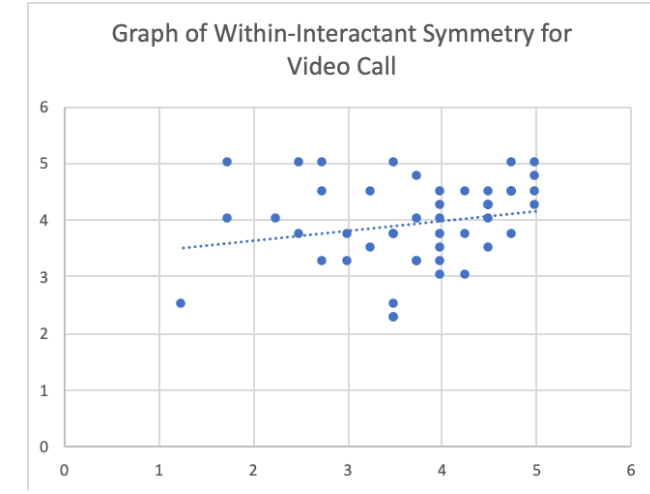

\subsection{Effects on Core Affect}

Research question two looked to compare emotions between the two conditions. Changes within the constructs of general positive affect and general negative affect across $3 \mathrm{D}$ and $2 \mathrm{D}$ conditions were compared. An independent-samples t-test was run to determine if there were differences in the mean changes between the $3 \mathrm{D}$ and $2 \mathrm{D}$ conditions. For the construct of general positive affect, the $3 \mathrm{D}$ condition elicit a lesser change in emotions $(\mathrm{M}=0.312, \mathrm{SD}=$ $0.44)$ than the $2 \mathrm{D}$ condition $(\mathrm{M}=0.3521, \mathrm{SD}=0.53)$, no statistically significant difference, $\mathrm{M}=-.04008$, $95 \%$ CI $[-0.2352,0.1955], \mathrm{t}(96)=-0.408, \mathrm{p}=.684$. For the construct of general negative affect, the 3D condition elicit a larger change in emotions $(\mathrm{M}=$ $0.1220, \mathrm{SD}=0.25)$ than the $2 \mathrm{D}$ condition $(\mathrm{M}=$ $0.0833, \mathrm{SD}=0.32$ ), no statistically significant difference, $\mathrm{M}=-.03867,95 \%$ CI [-0.1544,0.0771], $\mathrm{t}(96)=-0.663, \mathrm{p}=.509$.

For the construct of valence, the $3 \mathrm{D}$ condition elicit a larger change in means $(\mathrm{M}=4.36, \mathrm{SD}=0.66)$ than the $2 \mathrm{D}$ condition $(\mathrm{M}=4.25, \mathrm{SD}=1.24)$, no statistically significant difference, $\mathrm{M}=0.11,95 \% \mathrm{CI}[-$ $0.163,0.383], \mathrm{t}(96)=0.799, \mathrm{p}=426$. For the construct of arousal, the $3 \mathrm{D}$ condition elicit no change in means $(\mathrm{M}=03.50, \mathrm{SD}=1.07)$ than the $2 \mathrm{D}$ condition $(\mathrm{M}=3.5$, $\mathrm{SD}=1.24)$, no statistically significant difference, $\mathrm{M}=$ $0.00,95 \%$ CI $[-0.464,0.464], \mathrm{t}(96)=0.00, \mathrm{p}=1$. For the construct of dominance, the 3D condition elicit larger means $(\mathrm{M}=3.22, \mathrm{SD}=0.82)$, than the $2 \mathrm{D}$ condition $(\mathrm{M}=03.00, \mathrm{SD}=0.97)$ no statistically significant difference, $\mathrm{M}=0.22,95 \%$ CI [-.138, 0.578 ], $\mathrm{t}(96)=1.219, \mathrm{p}=0.226$.

\subsection{Effects on Novelty}

Research question three explored the construct of novelty of interactions between the $3 \mathrm{D}$ and $2 \mathrm{D}$ conditions. Within the overall construct of novelty, measurements of novelty experience, technology novelty, and novelty of communication were observed. For novelty of experience, an independentsamples t-test was run to determine if there were differences between the $3 \mathrm{D}$ and $2 \mathrm{D}$ conditions. The $3 \mathrm{D}$ condition elicit a larger experience of novelty $(\mathrm{M}=$ $4.56, \mathrm{SD}=0.71)$ than the $2 \mathrm{D}$ condition $(\mathrm{M}=3.81, \mathrm{SD}$ $=1.08)$, there was a statistically significant difference, $\mathrm{M}=0.748,95 \%$ CI $[0.3822,1.1128], \mathrm{t}(96)=4.062, \mathrm{p}$ $>0.001$. For novelty of technology, an independentsamples t-test was run to determine if there were differences between the 3D and 2D conditions. The 3D condition elicit a larger feeling of novelty of the technology $(\mathrm{M}=4.30, \mathrm{SD}=0.8391)$ than the $2 \mathrm{D}$ condition $(\mathrm{M}=3.67, \mathrm{SD}=1.1730)$, there was a statistically significant difference, $\mathrm{M}=0.633,95 \% \mathrm{CI}$ $[0.22566,1.041], \mathrm{t}(96)=3.084, \mathrm{p}>0.003$. For novelty of communication, an independent-samples t-test was run to determine if there were differences between the 
$3 \mathrm{D}$ and $2 \mathrm{D}$ conditions. The $3 \mathrm{D}$ condition elicits a larger feeling of novelty as a way to communication $(\mathrm{M}=4.40, \mathrm{SD}=0.8571)$ than the $2 \mathrm{D}$ condition $(\mathrm{M}$ $=4.06, \mathrm{SD}=1.0398)$, there was no statistically significant difference, $\mathrm{M}=1.921,95 \%$ CI $[-0.0439$, $0.7189]$, t $(96)=3.084, p=0.003$. For a summary of findings, refer to Table 4.

Table 4. Novelty significance

\begin{tabular}{|l|l|}
\hline Novelty Category & $\mathrm{p}$-Values \\
\hline $\begin{array}{l}\text { This technology was a } \\
\text { novel experience. }\end{array}$ & $\mathrm{p}<.010$ \\
\hline $\begin{array}{l}\text { This technology was } \\
\text { "new" or "refreshing." }\end{array}$ & $\mathrm{P}<0.01$ \\
\hline $\begin{array}{l}\text { This technology was a } \\
\text { novel way to } \\
\text { communicate. }\end{array}$ & $\mathrm{p}=0.479$ \\
\hline
\end{tabular}

\section{Discussion}

The focus of the first research question was to build an understanding of social presence around 3D communication. Results found that the degree of social presence rose or at least the facet co-presence rose. Co-presence is the degree to which others feel more aware and detected within an environment. The assumption is that the experimental 3D condition increased the degree of social presence by satisfying the first level within The Networked Minds Inventory. The rise in social presence is slight, as there were mixed correlations and no significance within the second level of psycho-behavioral accessibility. However, this may be task-dependent.

This experiment also focused on performance feedback, which limited the communication context. Social presence rose; however, there was no significance within emotional interdependence between the two conditions. This means our CMC types did not maximize or minimize social presence effect on emotions. This was additionally validated with the other metrics of the PANASX, valence, arousal, and dominance scales. The experiment showed how changes in general positive $\&$ negative affect are present, yet they are not significant. Valence, arousal, and dominance also exhibited changes, but when statistically compared, there was no significance across the 2D and 3D conditions.

Despite this, psycho-behavioral accessibility, attentional engagement and emotional interdependence display higher correlations of 3D than 2D. Attentional engagement measure behavior and responses to attention. The assumption is that within a 3D environment the participant was more attentive, not significantly different.
The results within the metrics of novelty support the $3 \mathrm{D}$ condition being significantly higher in the experience and the technology itself. However, novelty as a form of communication resulted in no significant difference between the conditions. This supports the notion that $3 \mathrm{D}$ communication is a new and novel experience and a "refreshing" technology; however, it is not a new form of communicating.

Cultural entertainment in science fiction, fantasy, or action stories have introduced holograms and 3D communication. Exploratory research suggests that science fiction media fuels the inspiration of interactive technologies [12], in addition to temporal context and product context [22]. The speculation is that multiple decades of cultural diffusion showing the form of 3D communication has decreased the novelty. Existing literature such as Marcus [17] and Mubin et al., [18] exhibits support for a novel technology to be inspired by science fiction. Our findings suggest that facets of novelty may be affected, but this is an assumption. Future research around this concept is necessary to make this assumption stronger and well supported.

\section{Conclusion}

Overall, within this context of research, there seems to be facets of social presence that display higher correlation within a 3D condition and other facets within a $2 \mathrm{D}$ conditions. There is a slight increase in the degree of social presence in the form of the perceptual level. Awareness and detection are substantially stronger within $3 \mathrm{D}$ condition than the 2D condition. Additionally, two facets of novelty of 3D were substantially different from $2 \mathrm{D}$. These facets being the experience of 3D communication and the perception that the technology is "new" and "refreshing."

Table 5. Lessons learned

\begin{tabular}{|l|l|}
\hline Concept & Lessons Learned \\
\hline Pocial & $\begin{array}{l}\text { Facets of social presence varied across the } \\
\text { different technology conditions; however, } \\
\text { the 3D condition had a significantly higher } \\
\text { level of perceptual awareness and } \\
\text { detection. Thus, raising the degree of } \\
\text { slightly social presence. }\end{array}$ \\
\hline Emotions & $\begin{array}{l}\text { Emotions may depend entirely on the } \\
\text { context of communication, or the task at } \\
\text { hand. There was no significant difference } \\
\text { between the two technology conditions. }\end{array}$ \\
$\begin{array}{l}\text { Additionally, whom one communicates } \\
\text { with may influence our emotions. }\end{array}$ \\
\hline Novelty & $\begin{array}{l}\text { Two dimensions of novelty were higher in } \\
\text { the 3D condition; these being the } \\
\text { experience, and the technology. However, }\end{array}$ \\
\hline
\end{tabular}




\begin{tabular}{|l|l|}
\hline & $\begin{array}{l}\text { the "form" of 3D communication was not } \\
\text { novel. This may give merit to the temporal } \\
\text { factor within novelty. }\end{array}$ \\
\hline
\end{tabular}

We understand that social presence is similar between video and hologram, while affect differs; however, one limitation was that the positive feedback given during the calls could have altered the participants' reactions. Second, the individual differences of the participants could account for the differences. Lastly, the nature of the experiment used trivial True/False questions.

In the future, research should change the type of feedback given. Future research should also consider the individual differences (e.g., the Big Five) and how they alter one's reaction to the technologies and social presence. Lastly, future research should also consider exploring different scenarios for the calls, such as solving a business problem or making a hiring decision. Additionally, future research should look to develop the communication context of groups of participants to understand mutual social presence. The Networked Minds Inventory was adapted within this research context to identify individual social presence. One future research idea is to incorporate a metric around the concept of propinquity, and the effect is may have on social presence and emotions. Communicating with a stranger in a laboratory setting gives some insight, yet stronger data may exist within field of measuring these concepts between kin or those with strong relationships.

Future research should use the shell of this experiment to identify and improve upon areas of interest. These areas of interest may change depending on context; however, the methodologies and analysis around social presence, emotions, and novelty were imperative to building an additional understanding of these concept's relationships within a telecommunication context. Furthermore, as telecommunication rapidly evolves, and attitudes change, it is important to understand psychological concepts, such as presence, emotions, and novelty to understand their effect on users. Understanding their relationships ultimately contributes to the "why" and the "how" they're adopted and used. Leading to advances and improved forms of communication.

\section{References}

[1] Agarwal, R., \& Prasad, J. (1997). The Role of Innovation Characteristics and Perceived Voluntariness in the Acceptance of Information Technologies. Decision Sciences, 28(3), 557582. https://doi.org/10.1111/j.15405915.1997.tb01322.x
[2] Biocca, F., Harms, C., and Burgoon, J. K. 2003. "Toward a More Robust Theory and Measure of Social Presence: Review and Suggested Criteria," Presence: Teleoperators and Virtual Environments (12:5), pp. 456-480. (https://doi.org/10.1162/105474603322761270).

[3] Biocca, F., Harms, C., and Gregg, J. 2001. "The Networked Minds Measure of Social Presence: Pilot Test of the Factor Structure and Concurrent Validity," East Lansing, MI: Media Interface and Network Design (MIND) Labs, Dept. of Telecommunication, Michigan State University, p. 9 .

[4] Bradley, M. M., \& Lang, P. J. (1994). Measuring emotion: The self-assessment manikin and the semantic differential. Journal of Behavior Therapy and Experimental Psychiatry, 25(1), 4959. https://doi.org/10.1016/0005-7916(94)900639

[5] Breazeal, C. 2003. "Emotion and Sociable Humanoid Robots," International Journal of HumanComputer Studies (59:1-2), pp. 119-155. (https://doi.org/10.1016/S1071-5819(03)00018$1)$.

[6] Collier, R. (2013). Optical Holography. Elsevier.

[7] Cronbach, L. J. (1951). Coefficient alpha and the internal structure of tests. Psychometrika, 16(3), 297-334. https://doi.org/10.1007/BF02310555

[8] Feldman, L. A. (1995). Valence Focus and Arousal: Individual Differences in the Structure of Affective Experiences. Journal of Personality and Social Psychology, 69(1), 153-166.

[9] Frank, B., \& Harms, C. (2002). Networked Minds Social

Presence Inventory: | (scales Only, Version 1.2) Measures of Co-Presence, Social Presence, Subjective Symmetry, and Intersubjective Symmetry.

[10] Geethanjali, B., Adalarasu, K., Hemapraba, A., Kumar, S. P., \& Rajasekeran, R. (2017). Emotion analysis using SAM (Self-Assessment Manikin) scale. Biomedical Research, $0(0)$. $\mathrm{http}: / / w w w . a l l i e d a c a d e m i e s . o r g / a b s t r a c t / e m o t i o n$ -analysis-using-sam-selfassessment-manikinscale-6115.html

[11] Izard, C. E. 1990. Basic Emotions, Relations Among Emotions, and Emotion-Cognition Relations, p. 5.

[12] Johnson, B. D. (2011). Science Fiction Prototyping: Designing the Future with Science Fiction. Synthesis Lectures on Computer Science, 3(1), 1- 
190.

https://doi.org/10.2200/S00336ED1V01Y201102 CSL003

[13] Kock, N. 2005. "Media Richness or Media Naturalness? The Evolution of Our Biological Communication Apparatus and Its Influence on Our Behavior Toward E-Communication Tools," IEEE Transactions on Professional Communication (48:2), pp. 117-130. (https://doi.org/10.1109/TPC.2005.849649).

[14] Lee, K. M. 2004. "Presence, Explicated," in Communication Theory, 14, International Communication Association, pp. 27-50.

[15] Lengel, R. H., and Daft, R. L. 1988. "The Selection of Communication Media as an Executive Skill," Academy of Management Perspectives (2:3), pp. 225-232.

(https://doi.org/10.5465/ame.1988.4277259).

[16] Lombard, M., \& Ditton, T. (1997). At the Heart of It All: The Concept of Presence. Journal of Computer-Mediated Communication, 3(2), 0-0. https://doi.org/10.1111/j.10836101.1997.tb00072.x

[17] Marcus, A. (2013). The History of the Future: Sci-Fi and Hci. Interactions, 20(4), 64-67.

[18] Mubin, O., Billinghurst, M., Obaid, M., Jordan, P., Alves-Oliveria, P., Eriksson, T., Barendregt, W., Sjolle, D., Fjeld, M., \& Simoff, S. (2016). Towards an Agenda for Sci-Fi Inspired HCI Research. Proceedings of the 13th International Conference on Advances in Computer Entertainment Technology - ACE2016, 1-6. https://doi.org/10.1145/3001773.3001786

[19] Neuliep, J. W. 2015. Intercultural Communication: A Contextual Approach, (6th ed.), SAGE Publications, Inc.

[20] Ramstad, E. (2011, January 3). Manufacturers Turn to Smart TV After 3-D Disappoints. Wall Street Journal. https://www.wsj.com/articles/SB1000142405274 8704835504576059783074010902

[21] Rice, R. E. (1992). Task Analyzability, Use of New Media, and Effectiveness: A Multi-Site Exploration of Media Richness. Organization Science, 3(4), 475-500. https://doi.org/10.1287/orsc.3.4.475

[22] Richins, M. L., \& Bloch, P. H. (1986). After the New Wears Off: The Temporal Context of Product Involvement. Journal of Consumer Research, 13(2), 280-285. https://doi.org/10.1086/209067
[23] Riva, G. 2002. "The Sociocognitive Psychology of Computer-Mediated Communication: The Present and Future of Technology-Based Interactions," CyberPsychology \& Behavior (5:6), pp. 581-598. (https://doi.org/10.1089/109493102321018222).

[24] Russell, J. A. (2003). Core affect and the psychological construction of emotion. Psychological Review, 110(1), 145-172. https://doi.org/10.1037/0033-295X.110.1.145

[25] Sherblom, J. C. 2020a. Computer-Mediated Communication Approaches and Perspectives, San Diego, CA: Cognella.

[26] Sherblom, J. C. 2020b. "Experience," in ComputerMediated Communication Approaches and Perspectives, San Diego, CA: Cognella, pp. 6394.

[27] Schnars, U., Falldorf, C., Watson, J., \& Jüptner, W. (2015). Digital Holography. In U. Schnars, C. Falldorf, J. Watson, \& W. Jüptner (Eds.), Digital Holography and Wavefront Sensing: Principles, Techniques and Applications (pp. 39-68). Springer. https://doi.org/10.1007/978-3-66244693-5_3

[28] Short, J., Williams, E., and Christie, B. 1976. The Social Psychology of Telecommunications, John Wiley and Sons Ltd.

[29] Tatikonda, M. V., \& Rosenthal, S. R. (2000). Technology Novelty, Project Complexity, and Product Development Project Execution Success: A Deeper Look at Task Uncertainty in Product Innovation. IEEE Transactions on Engineering Management, 47(1), 74-87. https://doi.org/10.1109/17.820727

[30] Watson, D., and Clark, L. A. 1999. "The PANAS-X," University of Iowa. (https://doi.org/10.17077/48vt-m4t2).

[31] Wells, J. D., Campbell, D. E., Valacich, J. S., \& Featherman, M. (2010). The Effect of Perceived Novelty on the Adoption of Information Technology Innovations: A Risk/Reward Perspective. Decision Sciences, 41(4), 813-843. https://doi.org/10.1111/j.15405915.2010.00292.x

[32] Yik, M., Russell, J. A., and Steiger, J. H. 2011. "A 12Point Circumplex Structure of Core Affect.," emotion (11:4), pp. 705-731. (https://doi.org/10.1037/a0023980). 